\title{
Classifying Execution Times in Parallel Computing Systems: A Classical Hypothesis Testing Approach
}

\author{
Hugo Pacheco, Jonathan Pino, Julio Santana, Pablo Ulloa, and Jorge E. Pezoa* \\ Departamento de Ingeniería Eléctrica and Center for Optics and Photonics (CEFOP) \\ Universidad de Concepción, Concepción, Chile \\ \{hpacheco,jopino, juliosantana, pulloag, jpezoa\}@udec.cl
}

\begin{abstract}
In this paper two classifiers have been derived in order to determine if identical computer tasks have been executed at different processors. The classifiers have been developed analytically following a classical hypothesis testing approach. The main assumption of this work is that the probability distribution function (pdf) of the random times taken by the processors to serve tasks are known. This assumption has been fulfilled by empirically characterizing the pdf of such random times. The performance of the classifiers developed here has been assessed using traces from real processors. Further, the performance of the classifiers is compared to heuristic classifiers, linear discriminants, and non-linear discriminants among other classifiers.
\end{abstract}

\section{Introduction}

Pattern recognition is a well-established research area that, in brief, takes groups of known patterns and abstracts their fundamental characteristics in terms of classes or clusters of data. Technically speaking, the stage where information is abstracted from the known-patterns is termed as the training process, and the known-patterns are called the training classes from the data sets. Based upon the information obtained during the training process, classifiers are next mathematically devised with the goal of determining, as accurate as possible, the class of a given sample data. This stage is called the classification process [1. Lately, classification as well as pattern recognition techniques have been employed in new application areas, such as parallel data processing, distributed data processing, network analysis, intrusion detection, customer analysis at communication service providers, etc. The common factor in all these areas is that it becomes mandatory to classify either the execution time of the applications executed on the system or the sojourn time of customers in the system, $[2,3,4,5,6]$.

For instance, in computer networks' intrusion detection, it becomes necessary to monitor the processing time taken by a packet-analyzer to break down the

\footnotetext{
* The authors thank Professors Rosa L. Figueroa and Mario R. Medina with Universidad de Concepción for their valuable comments and suggestions. J. E. Pezoa acknowledges support of CEFOP and Grant CONICYT PFB-0824.
} 
data packets present in the computer network. If an accurate estimate of such processing time is at hand, the behavior of normal as well as abnormal packets flowing in the network can be understood, and more importantly for classification and pattern recognition purposes, such behavior can be abstracted in a mathematical fashion [2,3, 5]. In parallel computing systems where servers are not homogeneous, as in the case of distributed computing clusters, tasks assigned to such systems must be smartly mapped onto servers so that a balanced allocation of the computing resources can be achieved. It is know that an efficient allocation of the computing resources in parallel and distributed computing systems depends vitally on an accurate knowledge about the execution time of the individual tasks forming an application [4.7]. For instance, Zhang and Figuereido employed in [4] a principal component analysis and $k$-NN classifiers to categorize the execution time of tasks being processed on a cluster of computers. In [6], Yang et al. proposed a classifier, based on the maximum-likelihood principle, to determine if the customers of a telecommunications service-provider are switching or not between different companies. To do so, they trained classifiers to learn the waiting time that different service providers offer to their customers.

In this paper, two classifiers have been developed in order to distinguish if identical tasks, which have been assigned to processors in a parallel computing system, are executed or not on different processors. The classifiers have been developed analytically using a classical hypothesis testing approach and the Bayesian as well as the Neyman-Pearson design criteria. Using traces taken from a real distributed system, a training process was conducted in order to characterize the a priori probability distribution functions (pdfs) of the random times taken by the processors to a serve task. As a result of the training process, the pdfs of the execution times were fitted and mathematically modeled. The performance of the classifiers developed here has been evaluated by categorizing real data. Further, the performance of our classifiers was compared to generic classifiers such as heuristic classifiers, linear discriminants, quadratic discriminants, and classifiers based on the Mahalanobis distance. Results have shown that the classifiers developed in this work consistently outperform those generic classifiers. At last, we comment that the motivation of this work is to implement the classifiers developed here in the distributed system shown in [7] with the goal of enhancing the load balancing algorithms of the system.

The rest of this paper is organized as follows. In Section 2 the empirical characterization of the pdf of the execution times is carried out as part of the training process. In Section 3 a Bayesian and a Neyman-Pearson classifiers are developed, and their performance in classifying the execution time of tasks is evaluated in Section 4. Our conclusions and future work are presented in Section 5.

\section{Problem Definition and Training Stage}

\subsection{Problem Definition}

Consider a parallel computing system based on two classes of processors. Suppose that atomic identical tasks are assigned to the processors in the system for their 
concurrent execution. Due to the nature computing systems, executing such tasks on any processor takes a random execution time, which depends solely on the processor where they are executed. This paper addresses the problem of (i) characterizing, for the purpose of designing classifiers, the processing times of these atomic tasks; and (ii) classifying a series of samples of the execution time of tasks in one out of the two classes of processors that are known to exist in the parallel computing system.

\subsection{Training Stage and Characterization of the Execution Times}

Statistical classification theory assumes that the pdf of the hypotheses are known it a priori. In order to obtain the pdf of processing times of the tasks forming the application being executed on the parallel computing system, we have conducted an experimental characterization of these random times by means of training data-sets of each class of processor in the system. First, we describe the classes of processors employed in the system. The first class of machines corresponds to tablet computers equipped with Crusoe processors. We name this class as it Crusoe and we also associate such machines, in the classification problem, with the null hypothesis, $H_{0}$. The second class of machines corresponds to laptop computers equipped with a Pentium 4 Mobile processors. We name this second class as $P 4 m$ and associate them with the alternative hypothesis, $H_{1}$. For training purposes, a total of 22026 samples of the execution times were used in the case of the Crusoe class, while 9805 samples of the execution time were used during the characterization of the execution time of the $P 4 \mathrm{~m}$ class.

During the training process, different probability distributions were fitted for the execution times. In order to determine the best fit, the minimum total square error criterion was employed. As a result, the Log-Normal distribution was selected as the best model for the pdf of the execution time of both machines. Fitted pdfs for different distribution functions can be observed in Fig. 1, while in Table 1 the total minimum square errors for each fitted pdf are listed. From these results, the pdf of the random variable $T_{i}, i=\{0,1\}$, which denotes the execution time of $i$ th hypothesis $H_{i}, i=\{0,1\}$, is mathematically described by the function:

$$
f_{T_{i}}\left(t ; \mu_{i}, \sigma_{i}\right)=\frac{1}{t \sigma_{i} \sqrt{2 \pi}} \exp \left(\frac{-\left(\ln (t)-\mu_{i}\right)^{2}}{2 \sigma_{i}^{2}}\right) .
$$

The parameters of each pdf estimated from the training data sets are: $\mu_{0}=$ $0.7203, \sigma_{0}=0.5459$ for the Crusoe class and $\mu_{1}=0.9760, \sigma_{1}=0.4599$ for the $P 4 m$ class.

\section{Classifiers Design}

\subsection{Bayesian Classifier}

To design a Bayesian classifier, first we must define both the costs associated to all the different classification errors and the rewards associated to correct 


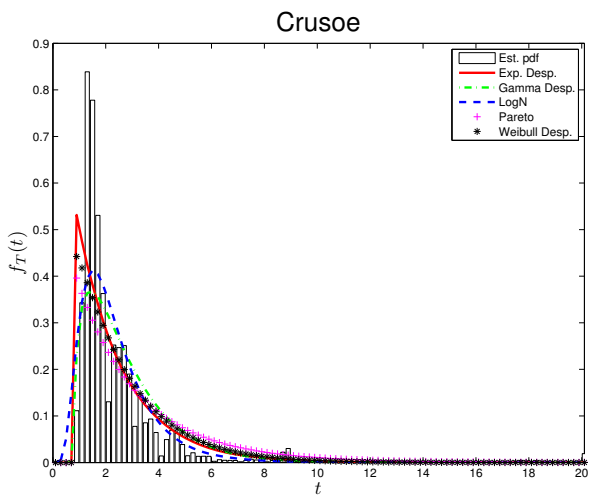

(a)

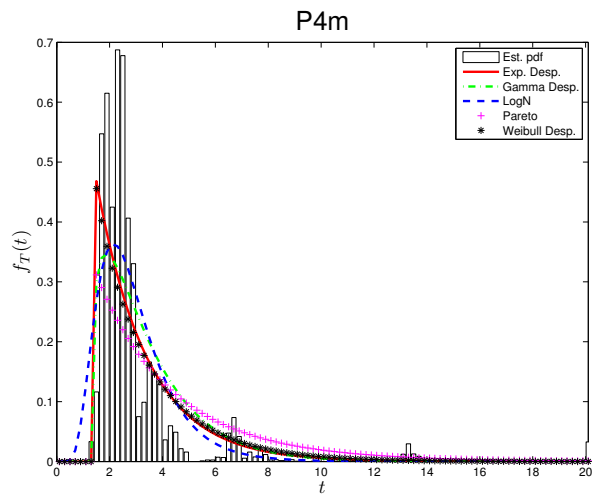

(b)

Fig. 1. Fitted pdfs for the execution time in: (a) Crusoe; and (b) $P 4 m$ processors

Tabla 1. Total square error between the fitted and the empirical pdfs of the execution time of tasks in the Crusoe class and in the $\mathrm{P} 4 \mathrm{~m}$ class of processors

\begin{tabular}{l|c|c|c|c|c}
\hline \hline & \multicolumn{4}{|c}{ Total square error } \\
Machine & Exponential & Gamma & Log-normal & Pareto & Weibull \\
\hline Crusoe & 0.612 & 0.524 & 0.466 & 0.698 & 0.603 \\
P4m & 0.610 & 0.566 & 0.524 & 0.826 & 0.656 \\
\hline \hline
\end{tabular}

classifications. It has been assumed here that the cost of making a mistake are symmetrical and unitary for the type I and type II errors and for both hypotheses, that is $c_{i j}=1, i, j=\{0,1\}, i \neq j$, while the rewards associated to correct classifications were assumed here to be zero, $c_{i j}=0, i, j=\{0,1\}$, $i=j$. In addition, it has been assumed here that the it a priori probabilities of the hypotheses $H_{0}$ and $H_{1}$ are identical and, as traditionally assumed, equal to 0.5. With this set-up defined, the classifier is designed after partitioning the sample set $\Omega=\Omega_{0} \cup \Omega_{1}$ by means of one or more threshold values, which must be determined in some optimal fashion with the goal of reducing the average classification risk that is denoted here as $\delta(\cdot)$. The minimal classification risk, $r^{*}(\delta)$, can be expressed as [8]:

$$
r^{*}(\delta)=\min _{\Omega_{1}}\{r(\delta)\}=\left\{t \in \Omega: \sum_{j=0}^{1} \pi_{j}\left(c_{1 j}-c_{0 j}\right) f_{T}^{j}(t) \leq 0\right\} .
$$

Once the probability distributions of the hypotheses, $f_{T}^{j}(t)$, the cost associated to each mistaken classificaion, $c_{i j}$, and the a priori probabilities, $\pi_{j}$, are known, the likelihood ratio test $(\mathrm{LRT}) L(t)=\frac{f_{T}^{1}(t)}{f_{T}^{0}(t)}=\frac{\sigma_{0}}{\sigma 1} \exp \left(\frac{\left(\ln (t)-\mu_{0}\right)^{2}}{2 \sigma_{0}^{2}}-\frac{\left(\ln (t)-\mu_{1}\right)^{2}}{2 \sigma_{1}^{2}}\right)$ can be used to find the threshold values defining the classification regions. 
The threshold values are obtained from the LRT by solving the second-order equation in the variable $z=\ln (t)$ :

$$
\left(\frac{\sigma_{1}^{2}-\sigma_{0}^{2}}{\sigma_{1}^{2} \sigma_{0}^{2}}\right) z^{2}+2\left(\frac{\mu_{1} \sigma_{0}^{2}-\mu_{0} \sigma_{1}^{2}}{\sigma_{1}^{2} \sigma_{0}^{2}}\right) z+\left(\frac{\mu_{0}^{2}}{\sigma_{0}^{2}}-\frac{\mu_{1}^{2}}{\sigma_{1}^{2}}-2 \ln \frac{\sigma_{1}}{\sigma_{0}}\right)=0,
$$

whose solutions are $t_{a}=2.027$ and $t_{b}=12.143$. These solutions uniquely define the following two classification regions: $\Omega_{0}=(0,2.027) \cup(12.143, \infty)$ and $\Omega_{1}=$ $[2.027,12.143]$.

\subsection{Neyman-Pearson Classifier}

The Neyman-Pearson criterion consists in maximizing the probability of true positive $P_{D}=P_{1}\left\{\Omega_{1}\right\}$, i.e, the probability of effectively announcing that the alternative hypothesis is observed when such hypothesis has truly occurred. The Neyman-Pearson criterion also states that the probability of true positive is maximized under the constraint of allowing a false positive or false alarm probability of $P_{F A}=P_{0}\left\{\Omega_{1}\right\}=\alpha$. To fulfill these requirements a partition $\Omega_{1}$ of $\Omega$ must be determined such that:

$$
\begin{gathered}
\left(\frac{\sigma_{1}^{2}-\sigma_{0}^{2}}{\sigma_{1}^{2} \sigma_{0}^{2}}\right) \ln ^{2}(t)+2\left(\frac{\mu_{1} \sigma_{0}^{2}-\mu_{0} \sigma_{1}^{2}}{\sigma_{1}^{2} \sigma_{0}^{2}}\right) \ln (t)+\left(\frac{\mu_{0}^{2}}{\sigma_{0}^{2}}-\frac{\mu_{1}^{2}}{\sigma_{1}^{2}}-2 \ln \frac{\sigma_{1}}{\sigma_{0}}\right) \geq \ln \gamma \\
\int_{\{t: L(t) \geq \gamma\}} f_{T}^{0}(t) \mathrm{d} t=\alpha
\end{gathered}
$$

where $\gamma$ is the threshold value associated to the LRT. Solving the system of non-linear inequalities (4) and (5) is a non trivial task, and to the best of our knowledge, it is not possible to solve for analytically. We have developed here an algorithm to solve numerically for this system of inequalities. The algorithm must execute the following steps:

1. An initial threshold value is set, say to $\gamma_{0}$.

2. Equation (44) is solved for to find the values $t_{a}\left(\gamma_{0}\right)$ and $t_{b}\left(\gamma_{0}\right)$. These solutions, which are parameterized by the initial threshold value, satisfy the LRT.

3. The false positive in the classification region $\Omega_{1}\left(\gamma_{0}\right)$ is calculated by means of the equation $\alpha\left(\gamma_{0}\right)=F_{0}\left(t_{b}\left(\gamma_{0}\right)\right)-F_{0}\left(t_{a}\left(\gamma_{0}\right)\right)$, where $F_{0}(\cdot)$ is the cummulative distribution function (CDF) of the null hypothesis.

4. If the relationship $\alpha\left(\gamma_{0}\right) \approx \alpha$ holds, then the classification region, $\Omega_{1}$, has been accurately defined, otherwise the algorithm must be executed from step 1 using a different threshold value.

In Fig. 2(a) the inequality (4) is shown. Note that if the false positive probability is fixed to $\alpha=0.5$, the values $t_{a}(\gamma)$ y $t_{b}(\gamma)$ satisfying the system of inequalities are $t_{a}(\gamma)=2.056$ and $t_{b}(\gamma)=11.97$. These values define the following classification regions: $\Omega_{0}=(0,2.056) \cup(11.97, \infty)$ and $\Omega_{1}=[2.056,11.97]$.

Note that by inspection of Figs. 2(a) and (b) a simple classification rule can be proposed. We propose here the boundary $t_{u}=2.0$, which approximately balances the areas under the pdfs of the execution times. This boundary and its classification regions, which have been determined in a qualitative and intuitive manner, are jointly termed here as the heuristic classifier. 


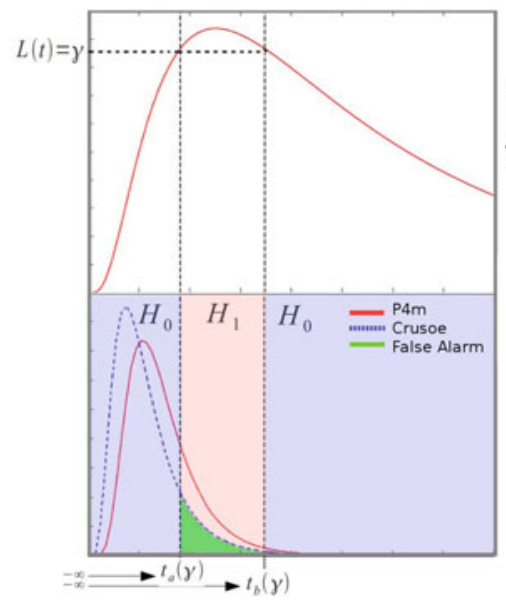

(a)

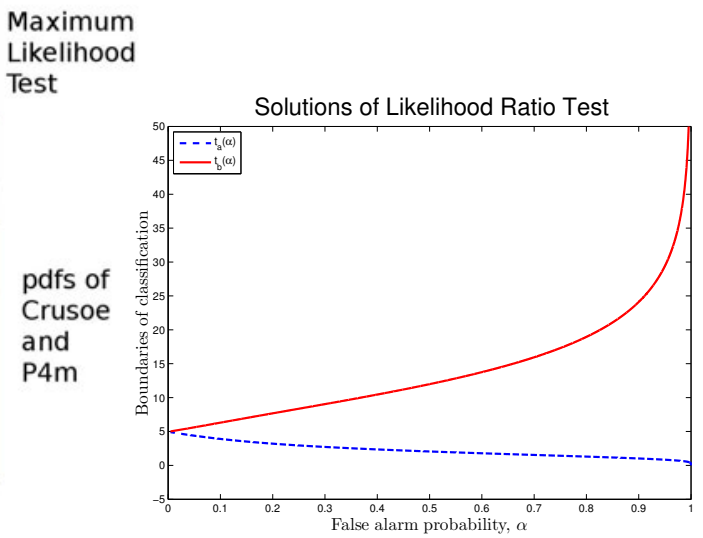

(b)

Fig. 2. (a) False positive probability $\alpha$, the LRT $L(t)$, and the pdfs of the execution times which represent the hypotheses of the problem. Solutions $t_{a}$ and $t_{b}$ of the LRT.

\section{Results}

To assess the performance of the classifiers devised here we have employed the following metrics: the percentage of hits, the percentage of positive classifications, the percentage of misclassification, the percentage of false positives, and classification error (in percentage). The metrics are mathematically defined as:

$$
\begin{aligned}
\% \text { Hits } & =100\left(\frac{Q\left\{H_{0}: H_{0}\right\}}{2 Q\left\{H_{0}\right\}}+\frac{Q\left\{H_{1}: H_{1}\right\}}{2 Q\left\{H_{1}\right\}}\right), \\
\% \text { Positive hits } & =100 \frac{Q\left\{H_{1}: H_{1}\right\}}{Q\left\{H_{1}\right\}}, \\
\% \text { False negative } & =100 \frac{Q\left\{H_{0}: H_{1}\right\}}{Q\left\{H_{1}\right\}}, \\
\% \text { False positives } & =100 \frac{Q\left\{H_{1}: H_{0}\right\}}{Q\left\{H_{0}\right\}}, \\
\% \text { Errors } & =1-\% \text { Hits },
\end{aligned}
$$

where $Q\left\{H_{i}\right\}$ is the number of times that the $i$ th class happen to occur during the assessment of the classifier, and $Q\left\{H_{i}: H_{j}\right\}$ is the number of times that the $i$ th class was announced as true, when actually the $j$ th class was the true class from which the sample under analysis was drawn.

The results of the classifiers developed in this paper are listed in Table 2. For comparison, we have employed the tool classify from Matlab's statistics toolbox®, and we have trained (using the same data employed to character- 
ize the distributions) classifiers based on linear discriminants, diag-linear discriminants, quadratic discriminants, diag-quadratic discriminants, and classifiers based on the Mahalanobis distance. The performance results of these Matalab based classifiers are listed in Table 3 .

From Table 2 we can observe that the Bayesian and the Neyman-Pearson classifiers achieve a fairly good percentage of hits. We note, however, that the Neyman-Pearson classifier offers some versatility over the Bayesian classifier through the false alarm parameter. The designer, or even the user, of a NeymanPearson classifier may adjust the false alarm probability to control the size of the classification regions. From Tables 2 and 3 it can be observed that the classifiers designed in this paper clearly outperform the classification methods implemented in Matlab's statistics toolbox in both metrics percentage classification hits and percentage of classification errors.

Figure 3(a) shows the percentage of hits of the Neyman-Pearson classifier as a function of the probability of false alarm. From the figure it can be observed the existence of a point where the percentage of classification hits is the same for both processors. At this point, the probability of false alarm is approximately 0.45. From Fig. 3(a) it can also be observed that the point where the percentage of classification hits is maximized corresponds to approximately to a probability of false alarm of 0.7. Figure 3(b) shows the receiver operating curve of the Neyman-Pearson classifier. From such figure we can observe that the marginal increment in the probability of classification is higher for values of the probability of false alarm below 0.5, as compared with the marginal increment observed for probabilities of false alarm larger than 0.6. In a more theoretical matter, it can be noticed that the receiver operating curve shown in Fig. 3.(b), which was calculated numerically, indeed exhibits the property of concavity expected from any receiver operating curve $[$ ]

Tabla 2. Performance of the classifiers designed in this paper

\begin{tabular}{l|r|r|r|r|r}
\hline \hline & Heuristic & Bayes & \multicolumn{3}{|c}{ Neyman-Pearson } \\
& & & $\alpha=0.2$ & $\alpha=0.5$ & $\alpha=0.7$ \\
\hline Hits & $58.84 \%$ & $66.05 \%$ & $52.80 \%$ & $66.04 \%$ & $67.57 \%$ \\
Errors & $41.15 \%$ & $33.95 \%$ & $47.20 \%$ & $33.95 \%$ & $32.43 \%$ \\
False alarm & $39.83 \%$ & $39.57 \%$ & $14.37 \%$ & $39.57 \%$ & $63.14 \%$ \\
Positive hits & $74.71 \%$ & $71.67 \%$ & $19.98 \%$ & $71.67 \%$ & $98.28 \%$ \\
False negative & $25.28 \%$ & $28.33 \%$ & $80.02 \%$ & $28.33 \%$ & $1.72 \%$ \\
\hline \hline
\end{tabular}

Tabla 3. Performance of generic classifiers

\begin{tabular}{l|c|c|c|c|c}
\hline \hline & Linear & Diag-linear & Quadratic & Diag-quadratic & Mahalanobis \\
\hline Hits & $57.08 \%$ & $57.08 \%$ & $58.25 \%$ & $58.25 \%$ & $32.58 \%$ \\
Errors & $42.92 \%$ & $42.92 \%$ & $41.75 \%$ & $41.75 \%$ & $67.41 \%$ \\
False alarm & $20.83 \%$ & $20.83 \%$ & $2.92 \%$ & $2.92 \%$ & $83.74 \%$ \\
Positive hits & $25.49 \%$ & $25.49 \%$ & $2.72 \%$ & $2.72 \%$ & $55.93 \%$ \\
False negative & $74.51 \%$ & $74.51 \%$ & $97.28 \%$ & $97.28 \%$ & $44.06 \%$ \\
\hline \hline
\end{tabular}




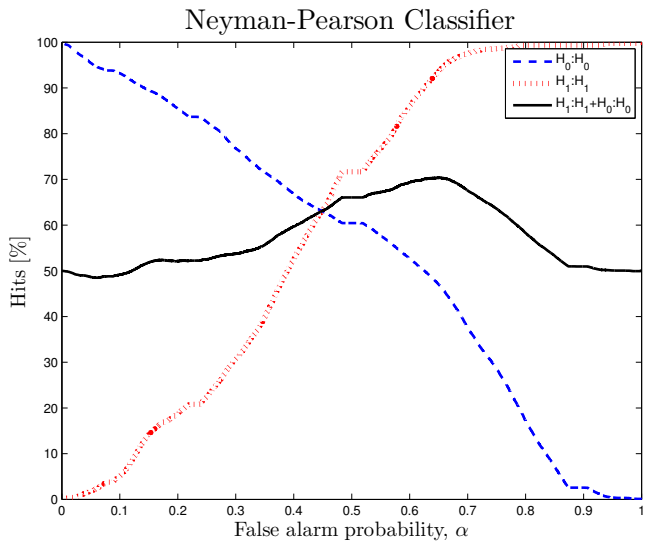

(a)

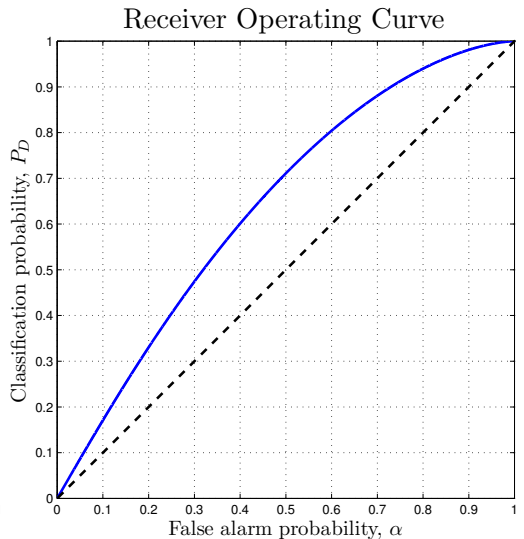

(b)

Fig. 3. Performance metrics of the Neyman-Pearson classifier: (a) Percentage of hits in classifying $H_{0}$ and $H_{1}$. (b) The receiver operating curve.

\section{Conclusions}

In this paper we have devised two classifiers for determining whether identical tasks have been executed or not in different processors. Since the Bayesiand and the Neyman-Pearson classifiers devised here have been developed for a specific application, their performance is indeed superior, as confirmed by our results, to the performance of generic classifiers which have been devised for classifying a wide class of applications. From our results we have observed also that the classification regions of Neyman-Pearson classifiers, with a probability of false alarm of 0.5, are similar to those obtained by the Bayesian classifier. This result is expected because a false alarm probability of 0.5 implies that the misclassification costs are symmetrical and unitary for both the null and the alternative hypothesis, and these costs are equal to the costs of the Bayesian classifier.

As a future work we will attempt to implement the classifiers developed here in a distributed system to improve its resource allocation algorithms.

\section{References}

1. Duda, R.O., Hart, P.E., Stork, D.G.: Pattern Classification, 2nd edn. WileyInterscience (November 2001)

2. Vasiliadis, G., Antonatos, S., Polychronakis, M., Markatos, E.P., Ioannidis, S.: Gnort: High Performance Network Intrusion Detection Using Graphics Processors. In: Lippmann, R., Kirda, E., Trachtenberg, A. (eds.) RAID 2008. LNCS, vol. 5230, pp. 116-134. Springer, Heidelberg (2008)

3. Dickinson, W., Leon, D., Podgurski, A.: Pursuing failure: the distribution of program failures in a profile space. In: FSE Conference Proceedings, pp. 246-255 (2001) 
4. Zhang, J., Figueiredo, R.J.: Application classification through monitoring and learning of resource consumption patterns. In: Proceedings of the 20th International Conference on Parallel and Distributed Processing, IPDPS 2006, Washington, DC, USA, p. 144 (2006)

5. Cabrera, J.B.D., Gosar, J., Lee, W., Mehra, R.K.: On the statistical distribution of processing times in network intrusion detection. In: Proc. 43rd IEEE Conference on Decision and Control (2004)

6. Yang, Y., Yang, Q., Lu, W., Pan, J., Pan, R., Lu, C., Li, L., Qin, Z.: Preprocessing Time Series Data for Classification With Application to CRM. In: Zhang, S., Jarvis, R.A. (eds.) AI 2005. LNCS (LNAI), vol. 3809, pp. 133-142. Springer, Heidelberg (2005)

7. Pezoa, J.E., Dhakal, S., Hayat, M.M.: Maximizing service reliability in distributed computing systems with random failures: Theory and implementation. IEEE Trans. Parallel and Dist. Systems 21(10), 1531-1544 (2010)

8. Kay, S.M.: Fundamentals of statistical signal processing: estimation theory. PrenticeHall, Inc., Upper Saddle River (1993) 